\title{
Error Estimates in Simple Quadrature with Voigt Functions
}

\section{By Alex Reichel}

In a wide range of spectral line computations occurring for example, in astrophysics and reactor physics, it is necessary to compute integrals of the form

$$
\int_{-\infty}^{+\infty} f(x) U_{0}(x, t) d x
$$

where

$$
U_{0}(x, t)=\frac{1}{(4 \pi t)^{1 / 2}} \int_{-\infty}^{+\infty} \frac{\exp \left[-(x-y)^{2} / 4 t\right] d y}{1+y^{2}}
$$

is the Voigt or Doppler broadened Breit-Wigner contour. Simple quadrature is often inaccurate due to the occurrence of steep and shifting plateaux in the integrand.

By using a modification of a method suggested by Goodwin [1] for integrals of the form

$$
\int_{-\infty}^{+\infty} f(x) \exp \left[-x^{2}\right] d x
$$

it is possible to estimate the error $E(h)$ in the quadrature formula

$$
\int_{-\infty}^{+\infty} f(x) U_{0}(x, t) d x=h \sum_{n=-\infty}^{+\infty} f(n h) U_{0}(n h, t)-E(h)
$$

where $h$ is the interval width. Without loss of generality, $f(x)$ may be taken as even.

As shown by the present author [2], for complex $z$,

$$
U_{0}(z, t)=\frac{-i}{(4 \pi t)^{1 / 2}} \int_{-\infty}^{+\infty} \frac{\exp \left[-u^{2} / 4 t\right](u-i) d u}{(u-i)^{2}-z^{2}}
$$

Consider the contour integral

$$
I=\oint \frac{f(z) U_{0}(z, t) d z}{\left\{1-e^{-2 \pi i z / h}\right\}}
$$

taken around the rectangle $-R \leqq x \leqq+R,-\pi / h \leqq y \leqq \pi / h$, where $h$ is sufficiently small as to include the poles of $f(z)$. Thus

$$
I=\frac{i}{(4 \pi t)^{1 / 2}} \int_{-\infty}^{+\infty} \exp \left[-u^{2} / 4 t\right](u-i) d u \oint \frac{f(z) d z}{\left[z^{2}-(u-i)^{2}\right]\left[1-e^{-2 \pi i z / h}\right]}
$$

giving

Received November 14, 1966. 


$$
\begin{aligned}
I= & h \sum_{n=-\infty}^{+\infty} f(n h) U_{0}(n h, t) \\
& +2 \pi i \sum_{k} \lim _{z \rightarrow z_{k}}\left\{\frac{\left(z-z_{k}\right) f(z)}{1-e^{-2 \pi i z / h}} \cdot U_{0}(z, t)\right\} \\
& +\frac{i}{(4 \pi t)^{1 / 2}} \int_{-\infty}^{+\infty} \exp \left[-u^{2} / 4 t\right]\left\{\frac{\pi i f(u-i)}{1-e^{-2 \pi i u / h-2 \pi / h}}-\frac{\pi i f(-u+i)}{1-e^{2 \pi i u / h+2 \pi / h}}\right\} d u
\end{aligned}
$$

where the $z_{k}$ are the poles of $f(z)$ within the rectangle.

Also, allowing $R \rightarrow \infty$,

$$
\begin{aligned}
I= & \int_{\infty+i \pi / h}^{-\infty+i \pi / h} \frac{f(x) U_{0}(x, t) d x}{\left[1-e^{-2 \pi i x / h}\right]}+\int_{-\infty-i \pi / h}^{\infty-i \pi / h} f(x) U_{0}(x, t) d x \\
& +\int_{-\infty-i \pi / h}^{\infty-i \pi / h} \frac{f(x) U_{0}(x, t) e^{-2 \pi i x / h} d x}{\left[1-e^{-2 \pi i x / h}\right]} .
\end{aligned}
$$

Now, if $\Gamma$ is the rectangle $-R \leqq x \leqq R,-(\pi / h) \leqq y \leqq 0$, we have for sufficiently small $h$ and $R \rightarrow \infty$,

$$
\begin{aligned}
\oint_{\Gamma} f(z) U_{0}(x, t)= & \frac{+i}{(4 \pi t)^{1 / 2}} \int_{-\infty}^{+\infty} \pi i f(u-i) \exp \left[-u^{2} / 4 t\right] d u \\
& +2 \pi i \sum_{k} \lim _{z \rightarrow z l k}\left\{\left(z-z_{l k}\right) f(z) U_{0}(z, t)\right\}
\end{aligned}
$$

where $z_{l k}$ are the poles of $f(z)$ in the lower half plane, poles on the $x$-axis requiring special treatment. Thus,

$$
\begin{aligned}
\int_{-\infty-i \pi / h}^{+\infty-i \pi / h} f(x) U_{0}(x, t) d x= & \int_{-\infty}^{+\infty} f(x) U_{0}(x, t) d x \\
& +\frac{i}{(4 \pi t)^{1 / 2}} \int_{-\infty}^{+\infty} \exp \left[-u^{2} / 4 t\right] \pi i f(u-i) d u \\
& +2 \pi i \sum_{k} \lim _{z \rightarrow 2}\left\{\left(z-z_{l k}\right) f(z) U_{0}(z, t)\right\}
\end{aligned}
$$

From (5), (6) and (8)

$$
\int_{-\infty}^{+\infty} f(x) U_{0}(x, t) d x=h \sum_{n=-\infty}^{+\infty} f(n h) U_{0}(n h, t)-E_{1}(h)-E_{2}(h)-E_{3}(h)
$$

where

$$
\begin{gathered}
E_{1}(h)=\frac{2 \pi}{(4 \pi t)^{1 / 2}} \sum_{r=1}^{\infty} e^{-2 r \pi / h} \int_{-\infty}^{+\infty} \exp \left[-u^{2} / 4 t\right] f(u-i) e^{-2 r \pi i u / h} d u \\
E_{2}(h)=2 \pi i \sum_{k} \lim _{z \rightarrow z l k}\left\{\left(z-z_{l k}\right) f(z) U_{0}(z, t)\right\} \\
\quad-2 \pi i \sum_{k} \lim _{z \rightarrow z_{k}}\left\{\frac{\left(z-z_{k}\right) f(z) U_{0}(z, t)}{1-e^{-2 \pi i z / h}}\right\}
\end{gathered}
$$

and 


$$
\begin{aligned}
E_{3}(h)= & \int_{+\infty+i \pi / h}^{-\infty+i \pi / h} \frac{f(x) U_{0}(x, t) d x}{1-e^{-2 \pi i x / h}} \\
& +\int_{-\infty-i \pi / h}^{\infty-i \pi / h} \frac{f(x) U_{0}(x, t) e^{-2 \pi i x / h} d x}{1-e^{-2 \pi i x / h}} .
\end{aligned}
$$

Now

$$
\begin{aligned}
E_{3}(h) & =2 \int_{-\infty-i \pi / h}^{\infty-i \pi / h} \frac{f(x) U_{0}(x, t) d x}{e^{+2 \pi i x / h}-1} \\
& =2 \sum_{r=1}^{\infty} \exp \left[-2 r \pi^{2} / h^{2}\right] \int_{-\infty}^{+\infty} f(u-i \pi / h) U_{0}(u-i \pi / h, t) e^{-2 r \pi i u / h} d u .
\end{aligned}
$$

For a wide class of functions $f(x)$ occurring in practice, the greatest value occurs at the origin, or the function is otherwise bounded within the range of integration.

A reasonable estimate might then be

$$
E_{3}(h) \doteqdot 2 f\left(\frac{i \pi}{h}\right) \sum_{r=1}^{\infty} \exp \left[-2 r \pi^{2} / h^{2}\right] \int_{-\infty}^{+\infty} U_{0}\left(u-\frac{i \pi}{h}, t\right) e^{-2 r \pi i u / h} d u .
$$

Values of $U_{0}(z, t)$ for complex $z$ are given in the author's paper [2]. We have for $-1<y<+1$,

$$
\begin{aligned}
U_{0}(x+i y, t) & =\frac{1}{2(1+y)} U_{0}\left(\frac{x}{1+y}, \frac{t}{(1+y)^{2}}\right) \\
+ & \frac{1}{2(1+y)} V_{0}\left(\frac{x}{1+y}, \frac{t}{(1+y)^{2}}\right) \\
+ & \frac{1}{2(1-y)} U_{0}\left(\frac{x}{1-y}, \frac{t}{(1+y)^{2}}\right)-\frac{i}{2(1-y)} V_{0}\left(\frac{x}{1-y}, \frac{t}{(1+y)^{2}}\right)
\end{aligned}
$$

and for $y>+1$

$$
\begin{aligned}
U_{0}(x+i y, t)= & \frac{1}{2(1+y)} U_{0}\left(\frac{x}{1+y}, \frac{t}{(1+y)^{2}}\right) \\
& +\frac{i}{2(1+y)} V_{0}\left(\frac{x}{1+y}, \frac{t}{(1+y)^{2}}\right) \\
& -\frac{1}{2(y-1)} U_{0}\left(\frac{x}{y-1}, \frac{t}{(y-1)^{2}}\right) \\
& -\frac{i}{2(y-1)} V_{0}\left(\frac{x}{y-1}, \frac{t}{(y-1)^{2}}\right),
\end{aligned}
$$

where

$$
V_{0}(x, t)=\frac{1}{(4 \pi t)^{1 / 2}} \int_{-\infty}^{+\infty} \frac{\exp \left[-(x-y)^{2} / 4 t\right] y d y}{1+y^{2}}
$$

is the Hilbert transform of $U_{0}(x, t)$. 
Thus, assuming $\pi / h>1$,

$$
\begin{aligned}
U_{0}(u-i \pi / h, t)= & U_{0}(-u+i \pi / h) \\
= & \frac{1}{2(1+\pi / h)} U_{0}\left(\frac{u}{1+\pi / h}, \frac{t}{(1+\pi / h)^{2}}\right) \\
& -\frac{i}{2(1+\pi / h)} V_{0}\left(\frac{u}{1+\pi / h}, \frac{t}{(1+\pi / h)^{2}}\right) \\
& -\frac{1}{2(\pi / h-1)} U_{0}\left(\frac{u}{\pi / h-1}, \frac{t}{(\pi / h-1)^{2}}\right) \\
& +\frac{i}{2(\pi / h-1)} V_{0}\left(\frac{u}{\pi / h-1}, \frac{t}{(\pi / h-1)^{2}}\right) .
\end{aligned}
$$

Substituting (17) in (13) and using the results

$$
\begin{aligned}
\int_{-\infty}^{+\infty} e^{-i p x} U_{0}(x, t) d x & =\pi \exp \left[-|p|-p^{2} t\right], \\
\int_{-\infty}^{+\infty} e^{-i p x} V_{0}(x, t) d x & =\pi i \exp \left[+p-p^{2} t\right], \quad p<0, \\
& =-\pi i \exp \left[-p-p^{2} t\right], \quad p>0, \\
& =0, \quad p=0,
\end{aligned}
$$

we find that the contribution to $E_{3}(h)$ from the first term in (17) for example is

$$
2 f\left(\frac{i \pi}{h}\right) \sum_{r=1}^{\infty} \exp \left[-2 r \pi^{2} / h^{2}\right] \cdot \frac{\pi}{2} \exp \left[-2 r \pi / h\left(1+\frac{\pi}{h}\right)-\frac{4 r^{2} \pi^{2} t}{h^{2}}\right] .
$$

For $h<\pi$ and $t>1$, this term is trivially small. Together with the three remaining terms of (17) in (13) we obtain four terms which cancel in pairs so that the value of $E_{3}(h)$ on this basis is zero.

For a similar class of functions $f(x)$, we have from (9),

$$
E_{1}(h) \doteqdot 2 \pi f(i) \sum_{r=1}^{\infty} \exp \left[-2 r \pi / h-4 r^{2} \pi^{2} t / h^{2}\right] \text {. }
$$

Only in special cases is this term important. The term $E_{2}(h)$ is readily computed using Eqs. (14) and (15). Tables and computer routines for the functions $U_{0}(x, t)$ and $V_{0}(x, t)$ are readily available.

Consider for example

$$
\int_{-\infty}^{+\infty} \frac{U_{0}(x, t) d z}{1+x^{2}}=\frac{\pi}{2} U_{0}\left(0, \frac{t}{4}\right)
$$

For the special case $t=4$, the value of the integral is 0.8570 to four places. Using an interval width $h=1$, we have

$$
U_{0}(0,4)+\sum_{1}^{\infty} \frac{2}{1+n^{2}} U_{0}(n, 4)=0.8612 .
$$

On account of the singularity in Eq. (18) we must use Eq. (9) to compute $E_{1}(h)$, and substitute 


$$
f(u-i)=\frac{1}{u^{2}+4}+\frac{i}{2 u}-\frac{i u}{2\left(u^{2}+4\right)} .
$$

Since

$$
\int_{-\infty}^{+\infty} \frac{\exp \left[-u^{2} / 4 t\right]}{u^{2}+4} e^{-2 r \pi i u / h} d u<\frac{1}{4}(4 \pi t)^{1 / 2} \exp \left[-4 r^{2} \pi^{2} t / h^{2}\right]
$$

and

$$
i \int_{-\infty}^{+\infty} \frac{\exp \left[-u^{2} / 4 t\right] u}{u^{2}+4} e^{-2 r \pi i u / h} d u<-\frac{i}{4}(4 \pi t)^{1 / 2} \exp \left[-4 r^{2} \pi^{2} t / h^{2}\right] \operatorname{erf}\left(\frac{i 2 r \pi \sqrt{ } t}{h}\right)
$$

both of which are trivially small for $h=1, t>1$, we see that

$$
E_{1}(h) \doteqdot \frac{+\pi^{2}}{(4 \pi t)^{1 / 2}} \sum_{r=1}^{\infty} e^{-2 r \pi / h} \operatorname{erf}\left(\frac{2 r \pi \sqrt{ } t}{h}\right) \doteqdot \frac{+\pi^{2}}{(4 \pi t)^{1 / 2}} \frac{1}{e^{2 \pi / h}-1} .
$$

For $h=1$ and $t=4, E_{1}(h) \doteqdot 0.0026$. Since $z_{l k}=-i, z_{k}=-i$, $+i$, Eq. (10) gives

$$
E_{2}(h)=\frac{2 \pi e^{-2 \pi / h}}{1-e^{-2 \pi / h}} U_{0}(i, t)=\frac{\pi}{2} \frac{U_{0}(0, t / 4)}{e^{2 \pi / h}-1} .
$$

For $h=1, t=4, E_{2}(h) \doteqdot 0.0016$.

Acknowledgment. The author thanks the Australian Institute of Nuclear Science and Engineering for a grant to cover the cost of computing.

University of Sydney

Sydney, Australia

1. E. T. Goodwin, "The evaluation of integrals of the form $\int_{-\infty}^{+\infty} f(x) e^{-x 2} d x$," Proc. Cambridge Philos. Soc., v. 45, 1949, pp. 241-245. MR 10, 575.

2. A. Reichel, "Voigt profile functions in the complex domain," J. Austral. Math. Soc., v. 4, 1964, pp. 476-488. MR 30 \#4007. 